\title{
Smart objects and wireless sensor networks for monitoring: sustainable technology in disaster management
}

\author{
V. Ferrara \\ Department of Information Engineering, Electronics and \\ Telecommunications, Sapienza University of Rome, Italy
}

\begin{abstract}
Disaster management needs to control significant data in the different phases: conditioning forecast from update in real time of event evolution, emergency plan, and first reconstruction of post event. At same mode, prevention activity consists primarily in the control of important quantities that characterize each event. The aim of paper is to examine two suitable methods of the monitoring for prevention and management of disaster: by means of sensor networks and drone or micro drone unmanned aerial vehicle (UAV). Differently from remote sensing, sensor networks are realized by distributing detection nodes on the territory. Each of two methods, sensor networks and with drone, shows peculiarities: sensor network is suitable for continuous monitoring and for forecasting; the monitoring that uses drones has more benefits in an emergency phase.

Keywords: smart objects, wireless sensor networks, low power, low voltage, unmanned aerial vehicle.
\end{abstract}

\section{Introduction}

Disaster represents generally the result of a chain of bad events. Prevention activity consists in the control of important quantities that characterize each event, in order to realize opportune measures against its occurrence.

At same mode, disaster management needs to control significant data in the different phases: conditioning forecast from update in real time of event evolution, emergency plan, and first reconstruction of post event. 
Disaster risk evaluation understood as forecast of a particular level of loss in terms of life, economic activity disrupted, people injured and so, is a complicated activity. Even the environmental phenomenon simpler is a function of more variables, often aleatory. Associated forecast model analyzes correlations of a selection of those variables and it simulates evolution of phenomenon. There are different software computer programs that realize models at different levels. From simplified version, faster, that uses few variables and produces coarse solutions, to sophisticated version, generally slower, that needs a lot of data inputs. Depending of simulated phenomena, some of these ones are data archived by historical acquisitions; others must be detected in real time. For example, a software simulation on forest fire propagation [1-4] includes historical data, as duration of dry conditions or as orographic slope, together with dynamical data, like speed and direction of wind. In another example, river flood and landslide [5-7], orographical data, climate characteristic of geographical area, profile and sections of rivers and flood plain are historical data, whereas e.g., the pluviometric measures are dynamical data. Both examples point out that all the data are necessary for the software tool is working properly.

Furthermore, real time data assume bigger importance when need monitoring areas of disaster in emergency management activity. In this case, the measure on demand of specific physical parameters and the snapshot observation of the overall effect of an event can modify boundary terms. That is consequence of new updated data: the software simulation evolves to different result and an operative decision is more suitable.

In the phase of the reconstruction, it asks that evaluations are global and detailed, but not necessary real-time. The globality is in order to detect the extension of damaged areas and to count the involved structures. Whereas, the detail represents the damage calculation, that can be estimated for single structure.

The aim of paper is to examine two suitable methods of the monitoring for prevention and management of disaster: by means of sensor networks and drone or micro drone UAV (Unmanned Aerial Vehicle). Differently from remote sensing, sensor networks are realized by distributing detection nodes on the territory. Extension of monitored area can't be too large if you want to limit node number and cost of full system. Each of two methods, sensor networks and with drone, shows peculiarities: sensor network is suitable for continuous monitoring and for forecasting; the monitoring that uses drones has more benefits in an emergency phase.

\section{Wireless sensor networks and smart objects}

The meaning of the terms smart objects and wireless sensor networks (WSN) is similar. A wireless sensor network is organized by small nodes that include: sensors (normally no actuators), wireless communication device, microcontroller and power source device. Main characteristic is to operate exclusively with wireless communication radio. Similarly, smart object indicates equipment that includes: one or more sensors/actuators, a tiny microprocessor (usually a 
microcontroller), a power source, and a communication device. Sensor and actuator allow interacting with the physical environment. The microprocessor /microcontroller elaborates data detected from sensors for storing or sending them. Power source supplies the total energy which is necessary for an efficient work of the node. The communication device enables the node to communicate measures of its sensor the outside, to a control station that collects full data from all the nodes, and receives input from other smart objects. But, unlike wireless sensor network, to implement the network, the communication device can use both technologies: wireless and wire. If the radio communication device is wireless, a more pervasive network can be built. So, smart objects and wireless sensor networks can be considered really network nodes, dedicated to monitoring. But, moreover, the previous definition shows how the smart objects have a greater interaction with the physical world if the devices are among them.

\subsection{IP smart objects}

Smart objects can use internet as communication middle. In this case its architecture is based on IP (Internet Protocol), and it can use UDP (User Datagram Protocol) or TCP (Transport Control Protocol) as transport protocol. UDP is the simplest protocol. It used, e.g., in a link where the transmitter does not have to wait for a response message by a receiver for confirming correct receipt. At the contrary TCP is a reliable byte stream. Twenty-seven companies founded a worldwide industry alliance to promote the usage of IP standard, open and interoperable, for smart objects. Cisco, Atmel, Arch Rock, Sun Microsystems, etc. have been founding members of this alliance, named IPSO (IP for Smart Object). An activity of IPSO is the production of white papers for divulging tutorial on technologies and use cases. In particular, the alliance spreads general characteristics of low-power technologies, embedded web services, smart cities, etc. Many WSN applications have been developed using internet for showing state and evolution of environmental quantities detected. So, web sites enable to consult and to input data that characterize far areas in the world. In order to develop these applications, it can use one among different operating systems for smart objects, like Contiki, TinyOS, FreeRTOS, that are all open sources. In particular, FreeRTOS has been designed for embedded systems and TinyOS for research into sensor network and smart objects. Furthermore, smart objects system implemented with web services need of lower complexity. Consequently it uses a light architecture model, as REST (Representational State Transfer).

\subsection{Non-IP smart objects}

The web service isn't the primary purpose of monitoring activities. Moreover, IP architecture is heavyweight in the case of low-power networks. That is, all networks built on the IEEE 802.15.4 standard protocol: a standard radio technology dedicated just for low-power, low-data-rate applications [8]. So, nonIP smart objects technologies are frequently used in the monitoring activities, specifically in the case of low-power, short-range, and low data rate. Proprietary 
protocol specifications for wireless communication based on IEEE 802.15.4 have been developed, as: ZigBee and Z-Wave. In particular, ZigBee is a network layer on top of the IEEE 802.15.4 standard, which includes PHYsical (PHY) and Message Authentication Code (MAC) layers. Radio frequency bands are in ISM (Industrial, Scientific and Medical) band: $2.4 \mathrm{GHz}$ with 16 channels for global use; $915 \mathrm{MHz}$ with 10 channels for few countries (North America, Australia, etc.), and $868 \mathrm{MHz}$ with one channel for EU countries. The $2.4 \mathrm{GHz}$ radio frequency allows a maximum data rate of $250 \mathrm{Kbits} / \mathrm{s}$. The band $915 \mathrm{MHz}$ permits a data rate of $40 \mathrm{Kbits} / \mathrm{s}$, and for $868 \mathrm{MHz}$ radio frequency the same data rate decreases to $20 \mathrm{Kbits} / \mathrm{s}$. Modulations are Bi-Phase Shift Keying (BPSK) for bands 868 and $915 \mathrm{MHz}$, and Offset quadrature phase-shift keying (OQPSK) for $2.4 \mathrm{GHz}$ radio frequency. Access mode is the Carrier Sense Multiple Access / Collision Avoidance (CSMA/CA): nodes occur if channel is free before transmission. But when transmission needs a low latency, the GTS (Guaranteed Time Slots) is the access mode.

Companies as: Texas Instruments, Microchip, Digi International, are building RF transceivers that are evolving to higher performances. For example, if you improve the sensitivity of the receiver, the budget of the link increases while always using the same transmitting power. Table 1 compares characteristics of three different radio frequency transceivers, and puts in evidence how area of their applications can be more extensive. Augmentation of link budget is to the detriment of power level, because its consumption increases, but fortunately with less strength if receiver sensitivity was raised. If maximum distance between two nodes can augment, then configurations number with which network can be designed increases too.

Table 1: $\quad$ Technical characteristics of three RF transceivers.

\begin{tabular}{|c|c|c|c|}
\hline & $\mathrm{CC2520}$ & MRF24J40MA & Xbee PRO SE \\
\hline Company & Texas Instruments & Microchip & Digi International \\
\hline Year & 2007 & 2008 & 2010 \\
\hline $\begin{array}{l}\text { Supported } \\
\text { protocols }\end{array}$ & ZigBee & $\begin{array}{c}\text { ZigBee, MiWi, } \\
\text { MiWi P2P and } \\
\text { Proprietary Wireless } \\
\text { Networking Protocol }\end{array}$ & ZigBee \\
\hline $\begin{array}{l}\text { Link budget } \\
\text { (line-of-sight) }\end{array}$ & 400 m@+5 dBm & $120 \mathrm{~m} @+5 \mathrm{dBm}$ & $\begin{array}{l}3200 \mathrm{~m} @+17 \mathrm{dBm} \\
1500 \mathrm{~m} @+10 \mathrm{dBm}\end{array}$ \\
\hline Supply range & $1.8 \mathrm{~V}-3.8 \mathrm{~V}$ & $2.4 \mathrm{~V}-3.6 \mathrm{~V}$ & $3 \mathrm{~V}-3.4 \mathrm{~V}$ \\
\hline Current RX & $\begin{array}{c}18.5 \mathrm{~mA} @-50 \mathrm{dBm} \\
\mathrm{RX}\end{array}$ & $19 \mathrm{~mA}$ (typical) & 45 mA@3.3V \\
\hline Current TX & $\begin{array}{c}25.8 \mathrm{~mA} @ 0 \mathrm{dBm}, \\
33.6 \mathrm{~mA} @+5 \mathrm{dBm}, \\
<1 \mu \mathrm{A} \text { power down }\end{array}$ & $\begin{array}{l}23 \mathrm{~mA} \text { (typical) } \\
2 \mu \mathrm{A} \text { sleep mode }\end{array}$ & $\begin{array}{l}295 \mathrm{~mA}-170 \mathrm{~mA} \\
3.5 \mu \mathrm{A} \text { power down }\end{array}$ \\
\hline Receiver sensitivity & $-98 \mathrm{dBm}$ & $-94 \mathrm{dBm}$ & $-102 \mathrm{dBm}$ \\
\hline RF frequency range & $2394-2507 \mathrm{MHz}$ & $2405-2480 \mathrm{MHz}$ & $2405-2480 \mathrm{MHz}$ \\
\hline
\end{tabular}

From the view point of sustainability, the total cost of a network node has decreased in recent years. Radio frequency transceiver cost is in the range from less than ten to forty Euros, functionally to required performance. The cost of a 
microcontroller is around a few tens of Euros. Sensors show a variable expense, depending on the type of measurement required, but it is decreasing little by little thanks to the development of MEMS (Micro Electro-Mechanical Systems) and embedded technologies. Many industries-leading supply sensors in the fields as: geo-mechanic, bioscience, flow-dynamic, meteorology, etc. So, you can realize different measurements and monitoring, by coupling sensors like: flow, tiltmeter, pressure, humidity, temperature, CMOS (Complementary Metal-Oxide Semiconductor) image sensors, CCD (Charge-Coupled Device), soil moisture, PH measurement, dissolved oxygen probe, water depth, water level, and so on. Therefore, based on wireless sensor networks or smart objects, the monitoring projects are a challenge whose resolution is currently efficient and in the future will be more convenient.

\subsection{Harvesting power supply}

In cost evaluation need include the maintenance item. For a sensor network, sites of the nodes should be selected for principally satisfying: area of interest for the measurement, and a radio link satisfying among other connected nodes. The requirement of nearness to public power line cannot limit the choice of location. The feature of low-power pushes to design of a power-supply device for smart object which uses harvesting and scavenging methods [9-12, 14]. Harvesting and scavenging convert power from human activity or limited energy from ambient. A definition considers harvesting the only method of conversion from ambient energy sources that are well characterized and regular. Some energy sources of interest for WSN are: ambient airflow, ambient light, ambient radio frequency, biologic, thermoelectric, and vibrational micro-generators. Performance of each conversion changes in a wide range, depending by type of ambient energy source and method adopted.

For ambient airflow source it can use microelectromechanical turbine which shows at 30 litres $/ \mathrm{min}$ performance of $1 \mathrm{~mW} / \mathrm{cm}^{2}$.

The available power from ambient light depends from the specific source (sun, incandescent bulb, compact fluorescent bulb, led) and from environmental condition, e.g. mid day with clear sky. Maximum available power is 100 $\mathrm{mW} / \mathrm{cm}^{2}$, if the source is the sun, and $1 \mathrm{~mW} / \mathrm{cm}^{2}$ at $3 \mathrm{~m}$ from a compact fluorescent bulb. Conversion efficiency of used technology decreases performance. For example, solar cells show a maximum conversion efficiency of 0.11 , if built by using a-Si or Dye-sensitized

The conversion from radio frequency energy uses a device named rectenna. This is a combination of antenna and rectifier [14]. But the radio frequency source doesn't assure sufficient power intensity for WSN nodes in the current technology. A simple example demonstrates this. Efficiency $\eta$ of rectenna device combines DC power to incident radio frequency, according to eqn (1). The Friis transmission eqn (2) evaluates the received radio frequency power $\left(P_{R}\right)$, when a transmitter at distance $R$ radiates a power $P_{T}$. In the eqn (2): $G_{T}$ and $G_{R}$ are gains with respect to an isotropic radiator respectively of transmitting antenna and 
receiving antenna, $\mu$ is the efficiency of transmitting antenna, and $\lambda$ is the wavelength of radio frequency.

$$
\begin{gathered}
\eta=\frac{P_{D C}}{P_{R F}}<1 \\
P_{R}=P_{T} \frac{G_{T} G_{R}}{\mu}\left(\frac{\lambda}{4 \pi R}\right)^{2}
\end{gathered}
$$

In the hypothesis of: isotropic radiators $G_{T}=G_{R}=1$, efficiency $\mu=1$, a relatively short distance $\mathrm{R}=10$, radiated power $\mathrm{P}_{\mathrm{T}}=1 \mathrm{~W}$, and radio frequency $\mathrm{f}=2.4 \mathrm{GHz}$, corresponding to $\lambda=0.125 \mathrm{~m}$ in free space, the value of maximum incident power is approximately $1 \mu \mathrm{W}$. This is a value too low. More energy can be harvested if device is hit from radio waves, numerous and outside the frequency band. BioElectrochemical System and in particular microbial fuel cell (MFC) exploits the properties of specific bacteria to produce electrons, by oxidation of organic compounds. An energy production continuous and unlimited has need that the bacteria are nourished, thus ensuring the survival of the bio-film, located at the anode of the cell. This makes these cells particularly interesting in applications devoted to monitoring of rural areas, land where there is plenty of nutritious food for the bacteria.

Thermoelectric conversion is obtained by means of a Carnot cycle. The temperature difference $\Delta \mathrm{T}$ limits the conversion efficiency. A thermoelectric generator consists of thermopile array. The most common material used to build the thermopiles is $\mathrm{Bi}_{2} \mathrm{Te}_{3}$, usable in the temperature range $0 \div 100{ }^{\circ} \mathrm{C}$. These generators produce a power of approximately $30 \mu \mathrm{W} @ \Delta \mathrm{T}=5{ }^{\circ} \mathrm{C}$.

Some vibrational micro-generators use piezoelectric material forming twolayer (on the top and down) bimorph, which are mounted as a cantilever beam. When you bend the beam down, a tension is present in the top layer and compresses the bottom layer. So, piezoelectric effect produces a voltage across each of the layers. The power output is maximized when the natural frequency of cantilever is equal to the driving frequency.

The choice of the harvesting device is made on the basis of the environment in which the system will have to work.

\subsection{Applications}

In previous articles [15] we described the use of WSNs for monitoring applied to the control of river floods. The WSN node included: PIC32MX360F512L microcontroller, MRF24J40MA IEEE 802.15.4 standard compliant radio frequency transceiver, OV7710 CMOS VGA colour CameraChipTM. Microcontroller and transceiver are Microchip devices. The single-chip video /imaging camera device is an Omnivision image sensor. MRF24J40MA is a PCB module (Printed Circuit Board) that includes an integrated PCB antenna.

The monitoring network included the placement of the sensors along the river at a distance less than the link budget transceiver. From Table 1 it is recognized that this distance is fixed at $120 \mathrm{~m}$, but it is preferred to place the sensors at a distance from each other of 30-40 m. If communication between two nodes fails, 
and the link to other no-contiguous nodes is on line of sight, this additional communication starts. In order to decrease energetic consumption of each node, a custom protocol has been used for this simplified mesh network.

At present, despite power consumption is greater, new generation of transceivers allows more flexible organization of the network mesh. Solar cells and water flow micro-turbine are suitable harvesting power sources for these network nodes. In the case of reduced production of energy for a period, e.g. solar cells during nocturnal hours, the harvesting device can be hybrid including energy storage in form of capacitor, or thin-film batteries.

In the last period we investigated the potential use of a different source of harvesting, like power supply of WSN communication node. Microbial fuel cell shows attractive qualities, in terms of eco-sustainability, low cost, and no maintenance for many years. MFC generates too low voltage, which must increase to suitable level, if it supplies energy to a WSN node, or smart objects. So, a charge pump must be included into the system design. Efficiency of cell depends from many factors. Mainly from terrain composition that should assure nutritious food for the bacteria. Investigation is in progress to verify how $\mathrm{pH}$ influences cell efficiency based on specific bacteria. In parallel an efficient lowpower communication design is developing for satisfying the compatibility with limited available power source.

\section{Unmanned aerial vehicle}

After a disaster, a natural catastrophe, frequently it is necessary to monitor a specific area for evaluating damages or make a more complicate detection. The activity can be dangerous and direct intervention of human operators is slow because of difficulties to move forward. Recent events, such as the earthquake that happened in Italy at Emilia Romagna region, have demonstrated the efficient use of unmanned aerial vehicles (UAV). Drone with a micro-camera onboard has allowed monitoring areas of destroyed buildings. It flew over rubble, and it recorded images, useful for succour activity. If the weight of drone is greater than a threshold, e.g. $20 \mathrm{~kg}$, it must observe the rules of flight control agency, like ENAV, including the communication of flight plan. If so, weight limit should be respected for allowing the flight always. In any case, the present micro-UAVs show a weight below $2 \mathrm{~kg}$, and sufficient autonomy for considering them adapted to monitoring in emergency phase. Furthermore, all devices onboard should be light sufficiently to allow a correct flight and adequate autonomy. Many sensors can be included onboard: VIS and IR image sensors, particulate sensor, and so on. Therefore, monitoring of a restricted area can be carried out by means of platform no expensive, based on micro-UVA. The system also supports emergency teams in their aid activity. At present, the cost of micro-UAV, like basis quadrotor model, with weight less than $1 \mathrm{~kg}$, is smaller than $300 \div 400$ Euros. Normally the basis model includes: motors, telemetry, GPS and landing gear. So, if it adds inexpensive special sensor devices for a customized monitoring with UAV, the full cost will be sustainable. 
In perspective the system is open to new useful applications. For example, studies are in progress on the possibility to locate buried and trapped people by using of ground penetrating radar (GPR). In particular, the research is including in the future activity of a working group in European concerted research action COST.

Ground penetrating radar [16] provides information about the different layers of soil and the existence of buried objects. Since GPR is electromagnetic energy and a material in the soil shows a particular electrical conductivity, it is subject to attenuation as it moves through the same material. GPR energy moves with velocity that depends from another characteristic: the dielectric constant. The higher the dielectric, the slower the radar wave moves through the medium. Moreover, a higher difference of dielectric constant between two contiguous materials, results in a stronger reflection. So, when the radar antenna moves along one direction, with a constant velocity, a detected object is shown in the radargram output as a diffraction hyperbola. Figure 1 shows radargram of data recorded by using the SIR-2000 ground penetrating radar system (Geophysical Survey Systems, Inc. (GSSI), North Salem, NH, USA) with a radar frequency of $900 \mathrm{MHz}$. Horizontal axis of the figure represents the position; vertical axis is the two-way travel time of radar wave. If dielectric constant of material is known, vertical axis can show the depth. In our experiment, a knapsack is buried into a dry sand soil. GPR has detected the object in form of diffraction curve characterized by geometrical parameters.

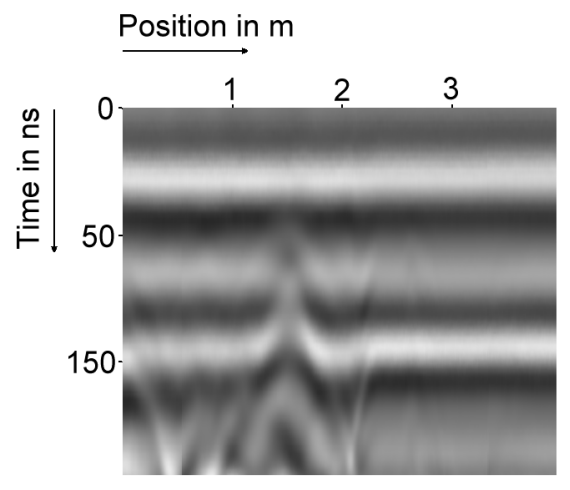

Figure 1: $\quad$ A radar profile recorded at $900 \mathrm{MHz}$.

\section{Conclusions}

We examined technologies adoptable for the monitoring that are useful for both activities: risk analysis together with its prevention activity, and disaster management. These new technologies are characterized by: more reduced dimensions than the past (nanometre-scale), and embedded systems more performing The improved characteristics are: light weight of final device, lowvoltage, low-power, and low-cost. The decreasing of power supply to lower 
value, but sufficient to operate the system properly, in all phases of its standard operation, adds freedom degrees to the design of a monitoring system. In the case of WSN and smart objects with wireless radio communication device, the location of a node is not imposed from electricity distribution network. Two features, i.e. low-power and light weight, result in more performing unmanned aerial vehicles that allow the monitoring of areas particularly dangerous. We examined some harvesting sources. And we selected the more suitable of these that should be used for designing smart objects and WSN. For the same reason, despite a relatively short link budget, IEEE 802.15.4 communication standard is the more appreciated. In accordance with these specifics we designed WSN to control risk of river flood. Our next researches will have two different themes: check the sustainable harvesting based on MFC, and investigate more valid setup of a GPR system for locating buried people.

\section{References}

[1] Van Wagner, C.E., A simple fire-growth model, The Forestry Chronicle, 45(2), 1969

[2] Rothermel, R.C., A mathematical model for predicting fire spread in wild land fuels (General Technical Report INT-115), Ogden, UT: USDA Forest Service, Intermountain Forest and Range Experiment Station. 1972

[3] Rothermel, R.C., How to predict the spread and intensity of forest and range fires (General Technical Report INT-143), Ogden, UT: USDA Forest Service, Intermountain Forest and Range Experiment Station, 1983

[4] Ferrara, V., Ottavi, C.M., Structures and organisation of an information tool dedicated to simulation and management of environmental risks, Proc. of conference Risk Analysis III, Ed. Brebbia, WIT Press, pp. 213-222, 2002

[5] Ferrara, V., Earth observation and network of in situ ground sensors for disaster management and early warning, Proc. of international Conference Disaster Management and Human health Risk: Reducing Risk, Improving Outcomes, Wit Press Southampton, pp. 3-12, 2009

[6] Cunge, J.A., Holly, F.M., Vernwey, A., Practical aspects of Computational River Hydraulics, Pitman, London, 1980

[7] Bates, P.D., Computationally efficient modelling of flood inundation extent, European Science Foundation Workshop, Bologna (Italy), Ed. BIOS, pp. 285-301, 2004

[8] Gutierrez, J.A., Naeve, M., Callaway, E. Bourgeois, M., Mitter, V., Heile, IEEE 802.15.4; A developing standard for low-power low-cost wireless personal area networks, IEEE Netw., September/October 2001, 15(5), pp. 12-19, 2001

[9] Paradiso, J. A., Starner, T., Energy scavenging for mobile and wireless electronics, IEEE CS and IEEE ComSoc, Pervasive computing, pp. 18-27, 2005

[10] Ottmann, G., Hofmann, H., Bhatt, A., Lesieutre, G., Adaptive piezoelectric energy harvesting circuit for wireless remote power supply, IEEE Trans. Power Electron., 17, pp. 669-676, 2002 
[11] Ungan, T., Reindl, L.M., Harvesting low ambient RF-sources for autonomous measurement systems, IEEE Int. Instrumentation and Measurement Technology Conference, Victoria, Vancouver Island, Canada, May 12-15, 2008

[12] Roundy, J.S., Leland, E.S., Baker, J., Carleton, E., Reilly, E., Lai, E., Improving power output for vibration-based energy scavengers, Pervasive Comput., 4, pp. 28-36, 2005

[13] Weddell, A.S., Harris, N.R. and White, N.M., Alternative energy sources for sensor nodes: rationalized design for long-term deployment, IEEE Int. Instrumentation and Measurement Technology Conference, Victoria, Vancouver Island, Canada, May 12-15, 2008

[14] Akkermans, J.A.G., van Beurden, M.C., Doodeman, G.J.N., Visser, H.J., Analytical models for low-power rectenna design, IEEE Antennas Wireless Propagation Lett., 4, pp. 187-190, 2005

[15] Ferrara, V., Wireless network sensors energy efficient for monitoring and early warning", Proceedings of international Conference Disaster Management II, Ed. Brebbia, WIT Press, pp. 235-244, 2011

[16] Daniels, D., (2 ${ }^{\text {nd }}$ ed). Ground, Penetrating, Radar, London, U.K., IEE, 2004 